\title{
Surgery following Extracorporeal Cardiopulmonary Resuscitation in a Patient with an Extracardiac Rupture of Valsalva Sinus Aneurysm
}

\author{
Jin Hong Wi, MD' ${ }^{1}$, Jeong-Sook Seo, $\mathrm{MD}^{2}$ \\ ${ }^{1}$ Department of Thoracic and Cardiovascular Surgery and ${ }^{2}$ Division of Cardiology, Department of Internal Medicine, Busan Paik \\ Hospital, Inje University College of Medicine, Busan, South Korea
}

\section{ABSTRACT}

The rupture of sinus of Valsalva aneurysm (SVA) into the pericardial cavity is extremely rare and fatal. A 52-year-old man presented with an abrupt onset of chest pain and dizziness. An echocardiography and a computed tomographic angiography revealed a giant aneurysm of the noncoronary sinus (NCS) (maximum, $70 \mathrm{~mm}$ ) and pericardial effusion in favor of tamponade with a moderate degree of aortic insufficiency. On the way to the operating room, he suffered cardiac arrest, and extracorporeal cardiopulmonary resuscitation (ECPR) was initiated immediately. While he was prepared for surgery, the patient was supported by extracorporeal life support (ECLS) until cardiopulmonary bypass (CPB) was initiated. The sinus defect was reconstructed using a Dacron patch, followed by resection of the aneurysm. ECLS and CPB were discontinued successfully in the operating room. This study reports a case of an extracardiac rupture of SVA and the successful completion of surgery following ECPR.

\section{INTRODUCTION}

A sinus of Valsalva aneurysm (SVA) is defined as a significant dilatation of the aortic wall located between the aortic valve and the sinotubular junction. Ruptured SVA is a rare disease that comprises $0.96 \%$ of all cardiac surgeries [Chu 1990]. The rupture into the pericardial cavity is exceptional and comprises only $1 \%$ of ruptured SVA cases [Ott 2006]. This study reports a very rare case of an extracardiac rupture of SVA and the successful completion of surgery following extracorporeal cardiopulmonary resuscitation (ECPR).

\section{CASE REPORT}

A 52-year-old man with a personal history of bronchial asthma presented at the emergency room complaining of

Received November 26, 2018; accepted April 4, 2019.

Correspondence: Fin Hong Wi, Department of Thoracic and Cardiovascular Surgery, Busan Paik Hospital, Inje University College of Medicine, 75 Bokji-ro, Busanjin-gu, Busan 614-735 Korea; 8251890 6834; fax: 82518911297 (e-mail: wiccy@hanmail.net). chest pain. The pain had suddenly developed 2 hours prior. Upon his arrival in the emergency room, his arterial blood pressure was $70 / 40 \mathrm{mmHg}$ and his heart rate was 70 beats/ minute. He was conscious and oriented, but complained of dizziness and nausea. A transthoracic echocardiography (TTE) documented SVA, a moderate degree of aortic regurgitation, and a pericardial effusion in favor of tamponade. Given these findings, a transesophageal echocardiography (TEE) and computed tomographic angiography (CTA) were carried out. They showed a giant aneurysm of the noncoronary sinus (NCS) (maximum, $70 \mathrm{~mm}$ ) that led to a marked compression of the right atrium (Figure 1).

Emergency surgery was scheduled. However, the patient arrested upon transfer to the operating room and required cardiopulmonary resuscitation (CPR). During CPR, extracorporeal life support (ECLS) was instituted via the left femoral artery and vein. The time interval from cardiac arrest to start of ECLS was approximately 15 minutes.

The patient was prepared for surgery while he was supported by ECLS. The procedure was done via a median sternotomy with a cardiopulmonary bypass (CPB). During the procedure, the surgeon encountered pericardium that was full of blood and fresh clots. Active bleeding from the perforated NCS aneurysm was also observed. Following cardioplegic arrest, the SVA and ascending aorta were opened. A large sinus defect (3 $\times 3 \mathrm{~cm}$ ) was located at the level of the giant NCS and its lower margin was noncoronary annulus (NCA). All of the aortic valve leaflets were intact but the noncoronary leaflet was prolapsed because there was no structure to support the NCA. A Dacron graft was sutured along the sinus defect involving NCA in its lower margin through an incision in the aneurysm and ascending aorta (Figure 2). After reconstruction of the defect, most

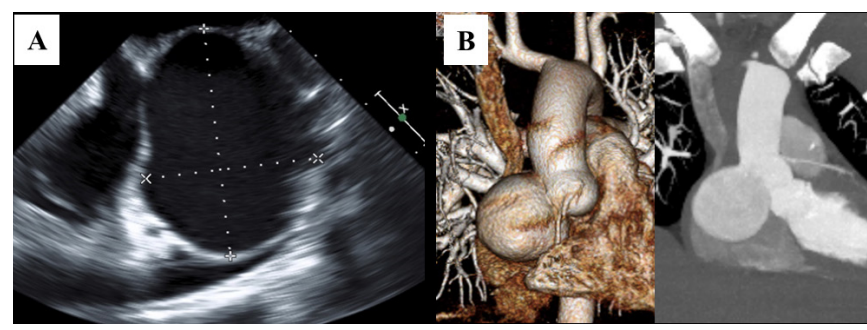

Figure 1. Preoperative imaging. (A) transesophageal echocardiography and (B) computed tomographic angiography (CTA) showing a giant aneurysm of the noncoronary sinus, leading to a marked compression of the right atrium. 


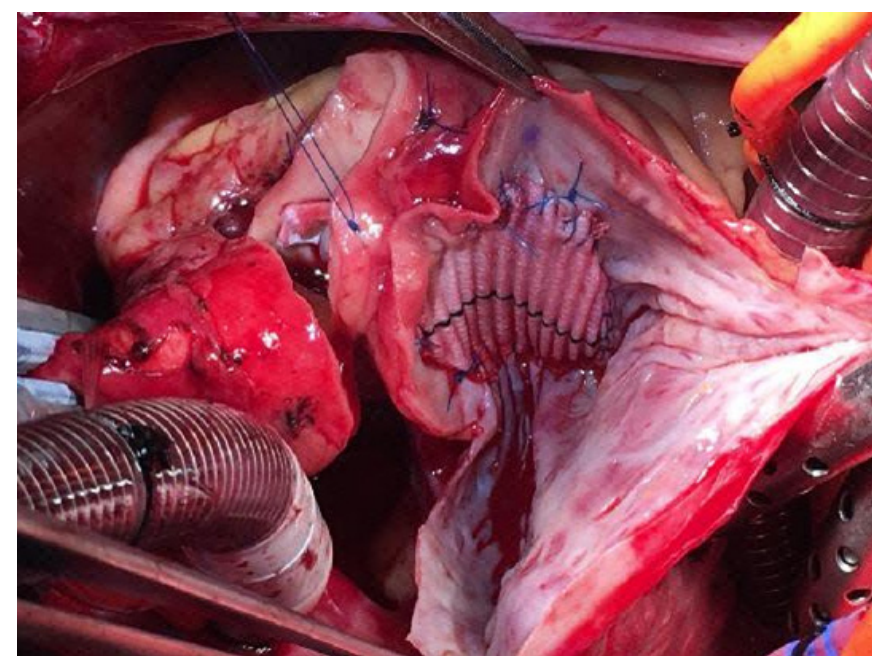

Figure 2. Intraoperative photograph. The sinus of Valsalva aneurysm and ascending aorta were opened. A large sinus defect $(3 \times 3 \mathrm{~cm})$ was closed with a Dacron graft via the incision of the aneurysm and ascending aorta.

of the aneurysm was resected, and the remnant portion of the aneurysm wall was approximated to cover the Dacron graft. The patient tolerated the procedure well and was discontinued from CPB and ECLS successfully at the end of procedure.

The patient made a full neurologic recovery and was discharged from the hospital after 12 days without any complications. A postoperative CTA showed an intact patch without aneurysm (Figure 3) and there was mild aortic insufficiency on postoperative TTE. At his last examination, two years after his surgery, the patient was in a good physical condition.

\section{DISCUSSION}

The incidence rate of SVA is low, accounting for $0.15-$ $1.5 \%$ of all cardiac surgeries [Ott 2006]. It generally affects the right sinus of Valsalva (94\% of cases) and the NCS (5\%); it rarely affects the left coronary sinus (1\%) [Ott 2006]. At the initial diagnosis, a rupture is found in $50-80 \%$ of patients with an SVA [Au 1998]. The majority of ruptures are in the cardiac chambers, resulting in aortocardiac fistulas. It occurs most frequently at the right ventricle followed by the right atrium, the left ventricle, and rarely the pericardium [Wang 2007]. Several cases of ruptured SVA into the pericardium have been reported, but few of the patients survived [Davarpasand 2014]. When cardiac arrest occurs, ECPR can be useful as it has a survival benefit over conventional CPR in patients with in-hospital cardiac arrest [Chen 2008]. This is the first report of a ruptured SVA into the pericardial cavity that was successfully managed by surgery following ECPR.

TEE is the diagnostic tool of choice. Associated with color Doppler, it precisely defines the location, morphology, size, associated lesions, and complications of the defect [Wang 2007]. Computed tomography demonstrates excellent anatomical depiction of the SVA and surrounding structures, identifies the ruptured SVA by depicting a jet

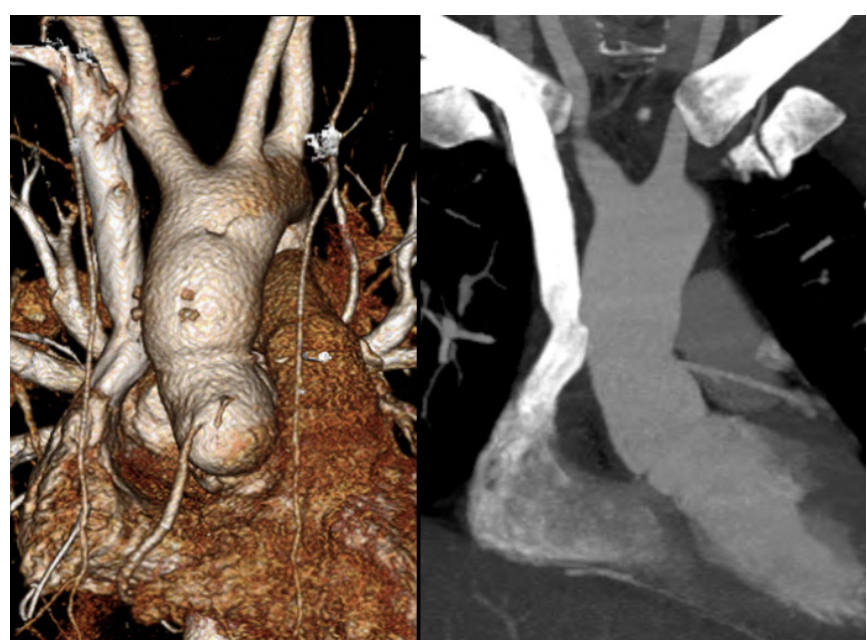

Figure 3. Postoperative imaging. The CTA showed that the repaired sinus of Valsalva was normal in size.

of contrast materials, and provides a comprehensive cardiac evaluation [Fathala 2012]. In the present case, we identified the patient's condition via echocardiography and CTA not only immediately but also exactly.

Extracardiac rupture of the SVA is extremely rare and produces hemodynamic instability due to cardiac tamponade. Thus, immediate diagnosis and emergency treatment are mandatory. ECLS and ECPR are advisable to save these patients.

\section{REFERENCES}

Buxton AE, Goldberg S, Harken A, Hirshfield J Jr, Kastor JA. 1981 Coronary-artery spasm immediately after myocardial revascularization: recognition and management. $\mathrm{N}$ Engl J Med 304:1249-53.

Buxton AE, Hirshfeld Jr JW, Untereker WJ, et al. 1982. Perioperative coronary arterial spasm: long term follow-up. Am J Cardiol 50:444-51.

Gaetano AL, Giulia Careri, Filippo Crea. 2011. Mechanisms of coronary artery spasm. Circulation 124:1774-82.

He GW, Taggart DP. 2016. Antispastic management in arterial grafts in coronary artery bypass grafting surgery. Ann Thorac Surg 102:659-68.

Lemmer JH Jr, Kirsh MM. 1988. Coronary artery spasm following coronary artery surgery. Ann Thorac Surg 46:108-15.

Marques KM, Jensen EE, Huybregts RA. 2006. Profound coronary spasm caused by a mediastinal draining tube. Heart 92:514.

Mayumi H, Kawachi Y, Kohno H, et al. 1994. Drug infusion through a branch of the aortocoronary vein graft for refractory coronary spasm. Ann Thorac Surg 54:869-71.

Pichard AD, Ambrose J, Mindich B, et al. 1980. Coronary artery spasm and perioperative cardiac arrest. J Thorac Cardiovasc Surg 80:249-54.

Schena S, Wildes T, Beardslee MA, Lasala JM, Damiano RJ Jr, Lawton JS. 2007. Successful management of unremitting spasm of the nongrafted right coronary artery after off-pump coronary artery bypass grafting. J Thorac Cardiovasc Surg 133:1649-50.

Teragawa H, Nishioka K, Fujii Y, et al. 2014. Worsening of coronary spasm during the perioperative period: A case report. World J Cardiol 6:685-8. 